\title{
Supervising Agent Team in an Agent-based Grid Resource Brokering System-Initial Solution
}

\author{
Wojciech Kuranowski \\ Software Development Department, Wirtualna Polska \\ Gdansk, Poland \\ Maria Ganzha, Marcin Paprzycki \\ Systems Research Institute Polish Academy of Science \\ Warsaw, Poland \\ \{maria.ganzha, marcin.paprzycki\}@ibspan.waw.pl \\ Ivan Lirkov \\ Institute for Parallel Processing, Bulgarian Academy of Science \\ Sofia, Bulgaria
}

\begin{abstract}
Currently, we are developing an agent-team based infrastructure for resource brokering and management in Grids. In this note we consider how team is supervised and how mirroring can be applied to improve chances of its long-term persistence.
\end{abstract}

\section{Introduction}

In our work we follow results presented in $[4,11]$ and develop a system in which software agents play role of resource brokers and managers in the Grid. To this effect, in [1] we have presented an overview of the proposed approach. In [2] we studied the most effective way of implementing yellow-page based matchmaking services. While in [6] we considered processes involved in agents seeking teams to execute their jobs, and in [9] we described processes taking place when agents seek teams to join. Finally, in [7] we have discussed trust-management-related issues. Since the proposed approach is based on agent teams, here, we discuss how to keep them together and prevent from being dissolved due to "technical difficulties" faced by team leader(s). We start from a brief overview of the proposed approach. Next, we discuss how a team leader controls status of its workers (their availability / existence). In the follow-up section we propose how mirroring can be applied to increase chance of team's survival.

\section{Proposed approach}

Rationale for the system is based on literature analysis and considering the Grid as an "open environment," (consisting of computers connected to the Internet [4]). A complete set of assumptions and earlier results can be found in $[1,2,6,9,7,5]$. Here, we start by presenting the Use Case diagram of the system (in Figure 1) and discussing most important (for this note) properties of the system.

The Client Information Center (CIC) agent plays the role of a central repository where information about all other agents is stored. It contains detailed information about teams that look for workers and teams offering to execute a job.Utilization of the CIC represents a "yellow page" based approach to matchmaking (see, [12] for critical analysis of possible approaches to matchmaking).

Let us now assume that the system is already running for some time, so that there exist at least some agent teams and their "advertisements" are posted within the CIC. The User can either try to contribute resources to the Grid or would like to utilize services available there. User who wants to contribute resources to the Grid communicates with its agent (the local agent LAgent that becomes a worker) and formulates conditions for joining a team. The LAgent requests from the $C I C$ list of agent teams that satisfy its joining criteria. Upon receiving such a list, due to trust considerations, 


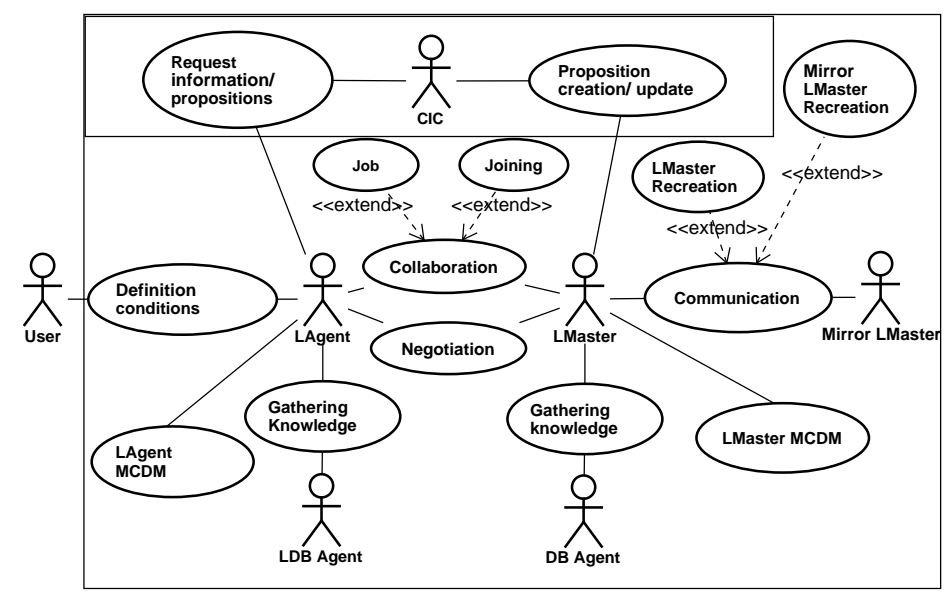

Figure 1. Use Case diagram of the proposed system

it may remove certain teams from the list. For all the remaining teams, the LAgent communicates with their LMasters utilizing FIPA Contract Net Protocol based negotiations [3] and multicriterial analysis [10] to evaluate obtained proposals. The result of interactions between the LAgent and LMasters may be twofold: (1) it finds a team to join, (2) no such team is found (either there were no offers, or they were unacceptable). In this situation the LAgent abandons the task and informs its User. In the case when the User requests that its LAgent arranges execution of a task, it specifies conditions of task execution. The LAgent queries the $C I C$ to find out which teams can do the job. Upon receiving a list of such teams, the LAgent removes from it teams that cannot be trusted. Next, it communicates with LMasters of the remaining teams and uses FIPA Contract Net Protocol and multicriterial analysis to find the best team to execute its job. If no team will satisfy conditions imposed by the User the LAgent reports this situation and awaits further instructions.

Let us now consider interactions between the LMaster and the LMirror. Both agents "mirror" eachother's existence and store information necessary to keep the team running, and if the LMaster fails, the LMirror can take its place. Recall that we assume that the proposed system works in an "open Grid," characterized by potential high volatility of its nodes. In such environment relatively frequent failures of LMasters have to be assumed. However, it has to be stressed that our goal is not creation of a "bullet-proof" environment. Rather, we are interested in providing an infrastructure with a reasonable level of resilience against common node failures.

Currently, we assume that the LMaster is the "founding father" of the team. Next, when the first agent joins its team, it automatically becomes its LMirror. Subsequent agents that join the team are informed by the LMaster which agent is the LMirror. In this way the LMirror becomes a trusted source of information (see below). Furthermore, the LMaster registers the LMirror with the CIC. Here, in the case of a crash of the LMaster the CIC has to know that a given LMirror has a "right" to represent its team and replace the failed agent. The LMaster and the LMirror share information that is pertinent to the existence of the team; e.g. list of workers and their characteristics, list of tasks that have been contracted to be executed, knowledge base that stores information about all past interactions with users and workers, etc. Now, it should be obvious why storing such information solely by the LMaster would mean that the team would vanish in the case its crash. In order to sustain the team, the LMaster and the LMirror check each-others existence regularly in short time intervals (see below). In the case when the LMaster crashes the LMirror takes over its role (becomes the new LMaster). The first action of the new LMaster is to promote one of worker agents to become its LMirror and pass to it all necessary information. Next, it informs all members of the team about its becoming the LMaster. Finally, it updates the team information with the CIC (informing who is the LMaster and the LMirror).

Similarly, upon finding that the LMirror agent is "gone," the LMaster immediately promotes one of workers to become the new LMirror and passes to it all necessary information. Next it informs the team as well as the CIC who is the new LMirror. Note that both the LMaster and the LMirror may crash "almost simultaneously" and thus the team will be "destroyed." 
However, at this stage of the development of the system we consider this outside of scope of our interests.

\section{Monitoring status of team workers}

As specified above, we assume that nodes in the Grid can disappear at any moment (e.g. a PC being turned off by a playing dog). Therefore, one of key functions of the LMaster is monitoring status of it team. The LMaster should also be able to evaluate the state of the network between itself and each worker. This latter knowledge can be used, among others, to allocate jobs and to adjust the monitoring procedure (see below). Note that while node crashes should be discovered very fast, the monitoring process should also avoid false positives (i.e. a short network outage should not be misconstrued as a node failure). Thus we have designed a monitoring system based on the LMaster "pinging" workers using minimalistic ACL messages. Specifically, each message consists of a string "ping" as its content, a reply-with field specifying the content of the response (string "re-ping"), and the reply-by field specifying the deadline for the response (if the message is obtained after the deadline passed, e.g. due to a network delay, the receiver will ignore it). After receiving a "ping-ACLmessage" worker replies to it.Upon receiving a reply, the LMaster knows that a given agent is alive. However, to be able to develop a robust, but flexible monitoring subsystem we have designed a somewhat more complicated approach than a simple ping-response. The screen-shot of the GUI of an LMaster (in Figure 2) is the basis of its description. We have to stress that this GUI has been used only for testing purposes. In actual runs, each LMaster obtains (as a text file) a set of parameters and acts autonomously without displaying results in a GUI. The monitoring process consists of the following steps (see Figure 2):

1. LMaster performs $X$ tests consisting of $Y$ pings each (both values $X$ and $Y$, as well as other parameters, are configurable and are "an input" to the LMaster; we assume that in the future, they will be autonomously adjusted depending on factors like: network conditions, trust etc.).

2. Each ping is send at a certain interval (specified in milliseconds). While we assume that pinging intervals for each agent may differ, currently a ping is send to all agents (message broadcast) and a response is expected within a specified time. The next ping is sent after a specified time has passed.

3. For each agent in the team, a response to a ping is a "pass" if it returns within a required time.
4. For a set of $Y$ pings a percent of failed attempts is calculated.

5. After $Y$ pings, the "fail-percent" value is compared with the allowed "fail-value" to establish if a given agent has passed one of $X$ tests.

6. After $X$ tests a total number of failed tests is found.

7. This value is compared with the number of tests that given agent was allowed to fail. Failure of a given number of test may (or may not) result in agent's removal from the team.

8. Counters are zeroed and the procedure is repeated.

In Figure 2 we can see the following values of the above defined parameters of the monitoring system:

- Ping interval: time between individual pings is set to $200 \mathrm{~ms}$ (the same value used for all agents)

- Max ping reply: for a given ping to be scored as a success, the response has to be received within 100 ms (part of the ACL-ping message; see above)

- Pings per test: each test consists of 20 pings

- Number of tests: each monitoring cycle consists of 15 tests

- Tests to pass: for the worker agent to be perceived as alive, it has to pass at least 4 tests (out of 15)

- Max loss: a test is passed if the total number of failed responses is smaller than $80 \%$ (for 20 pings per test, at least 16 have to be passed)

In Figure 2 we can also see that the LMaster is managing 5 agents (bottom left "sub-panel"), while data concerning results of a current liveliness test for the agent Worker2@home:1099/JADE is depicted in right top "sub-panel." Specifically, we can see that:

- Sent $=18$ pings have been send

- Received $=18$ responses have been received

- Loss $=0 \%$ losses were recorded (no losses)

- $M i n=1 \mathrm{~ms}$ was the fastest response to a ping

- $A v g=5 \mathrm{~ms}$ was the average response time

- $M a x=41 \mathrm{~ms}$ was the longest response time

- Test no. $=12 / 15$; test $12($ of 15$)$ is in progress

- Passed = 11 tests have been passed thus far (all tests, as test 12 is in progress) 


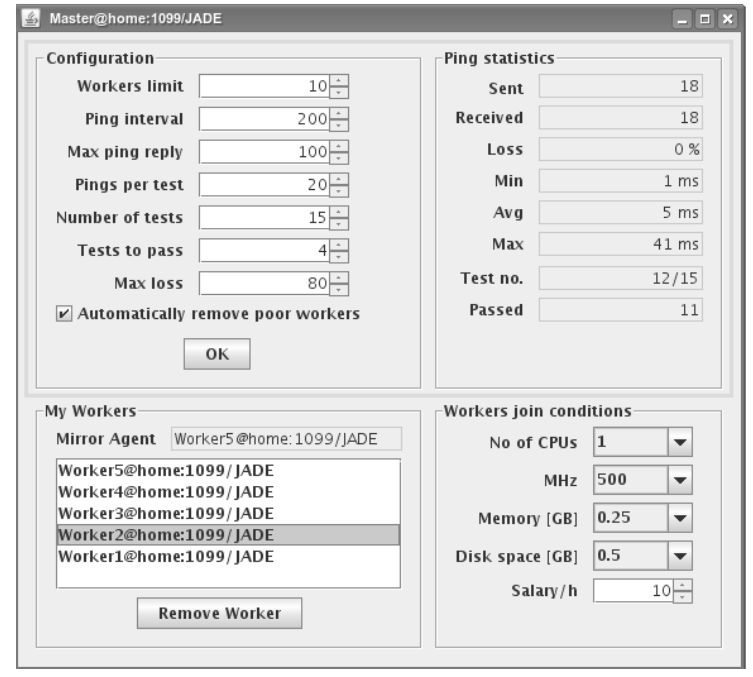

Figure 2. GUI illustrating monitoring of a team member by an LMaster

The proposed monitoring procedure is quite flexible. First, let us consider a "local Grid" (e.g. Grid nodes are in the same laboratory). Here, pings can be send often, expected response time can be short, while non-responsive nodes can be immediately removed (see the Automatically remove poor workers checkbox). The situation changes on the Internet. First, network outages may (should?) not result in immediate removal of a team member. It is even possible to not to remove any team member, but try to reach it/them, until connection is reestablished (note that this approach can be related directly to trust management, where some nodes are worthy our trust and we try to reach them, while some are not [7]). Furthermore, it is possible to relax selected parameters to avoid unnecessary team member removal. For instance, a number of passed pings within a round of testing can be reduced down to a single passed test (indicating that a node is actually alive).

Note also that testing parameters should be adjusted to the network conditions. For instance, if a packet loss rate is about $70 \%$, then monitoring cannot assume better sucess rate of pinging. Similarly, if the response time is about $100 \mathrm{~ms}$, the monitoring procedure has to take this into account. While adaptability of monitoring is not yet implemented, it is easy to see why network conditions may result in testing individualized for each team member. For instance, after each failed test-round probing tests are performed to match the changing network conditions (similarly to the RealPlayer adjusting settings to improve quality of sound).

\section{LMaster-LMirror interactions}

\subsection{Re-creating crashed LMirror or LMaster}

Monitoring is used also to find out when either the LMaster or the LMirror crash and have to be replaced/recreated. Replacing the LMirror is relatively simple. The LMaster uses monitoring to check liveliness of all team members, including the LMirror. If the LMirror fails the test it has to be recreated. When a team that is working already for some time, the LMaster has data about performance (as well as resources) of all workers and can use it to select the best agent to become the next LMirror. Obviously, the new LMirror should have a "non-stop contract" with the team (see [9]), a very fast network connection and appropriate resources. Promotion of a node to an LMirror proceeds in the same way as creation of the first LMirror; appropriate modules are send to it to be loaded, followed by all "mirror-data." Finally, the LMaster informs the team and the CIC, which agent became the new LMirror. During this process the LMaster stops responding to queries and performing any other tasks, as creation of an LMirror has the highest urgency (without its successful completion the team may disappear). Here, we omit the question: what happens if a high-quality node does not exist in the team, as actually not much can be done and such situation is very likely going to, sooner or later, result in team destruction. Furthermore, for the time being we 
omit considerations involved in the economical model of being an LMaster (i.e. not doing any actual work) and an LMirror (which can do some work, but also has to perform "duties" of the mirror, which are of higher priority than completing other tasks). These questions, while very interesting, require a comprehensive solution and are outside of the scope of this note.

When the LMaster crashes and the LMirror has to become a new LMaster we deal with a slightly more complicated situation. First, we need to establish how the LMirror finds out that there are problems with the LMaster. Obviously, the simplest solution would be if the LMirror would listen to ping-tests arriving from the LMaster and their disappearance would mean that the LMaster has crashed. In this case the LMirror could contact the AMS agent (FIPA mandated agent that, among others, manages white-page information about agents in the system; $A M S$ agent is provided by the JADE agent environment used in our work [8]) to check if the LMaster is still registered with the system (the $A M S$ agent automatically deregisters defunct agents) and use this information to start the LMaster recreation. However, this approach will not work as we assume that the LMaster should be able to adaptively adjust ping intervals, (e.g. based on the state of the network). Therefore, the interval between pings may change and the LMirror cannot use the "current ping rate" to correctly assess the state of the LMaster. Therefore, we have decided to utilize the same monitoring function used by the LMaster to monitor team members, for the LMirror to monitor state of the LMaster (however, the LMirror monitors only a single agent). After the LMaster stopped responding to test-pings the LMirror contacts the AMS agent and checks if the LMaster is still alive. If it is, it tests its existence again to prevent possible conflicts arising when two agents with the role LMaster are created in a single team. If the AMS agent confirms that the LMaster is gone, the following replacement procedure is initiated:

1. The LMirror creates a skeleton agent and loads it with LMaster modules. Specifically, the LMirror requests that the $A M S$ creates an agent based on a class that implements an LMaster agent. Here, we assume that it is easier for the LMirror to create a new agent, rather than modify itself. Note that there is a large difference between functions performed by the LMaster and the LMirror agents. For instance, the LMirror has only to monitor existence of the LMaster and "step-in" when it crashes (separately it executes user-jobs assigned to it by the LMaster). At the same time the LMaster is responsible for "keeping the team alive," contacts with clients and potential workers, etc.
2. The LMirror passes to the new LMaster the last version of the team-persisting data and awaits a confirmation that the data has been successfully replicated and assimilated (i.e. it awaits information that the new LMaster is fully functional).

3. Upon reception of the required confirmation, the LMirror informs all members of the team that a new LMaster has been created and will take over management of the team. Note that team members have been informed that the LMirror has a "right" to do so, as it can replace the LMaster if necessary. Separately, the LMirror informs the CIC about the change in team leadership (the $C I C$ is also ready for such a message to arrive, see above).

4. The current LMirror self-destructs as it is no longer needed, while the new LMaster takes over. Its first function is re-creation of an LMirror (utilizing possessed information about the existing team members). This is the same procedure as when the LMirror crashes. When the LMirror is fully re-created and data replicated to it, the new LMaster informs team members and the CIC which agent is the new LMirror of the team. Next, the new LMaster starts managing the team and interact with incoming messages from both team members and "outside agents." Note that completion of restoration of the LMaster + LMirror pair is the task that has the highest priority.

5. The new LMaster has to pass its current task (recall that the LMirror is executing clients' tasks) to a worker, as it will not be able to complete it (the LMasters works only on team-management related tasks). Furthermore, it may need to re-allocate the task being executed by the new LMirror to another agent (e.g. a high priority task that should not be interrupted by mirroring related activities).

6. Upon reception of a message from the LMirror, team members verify that the old LMaster is actually no longer available (by contacting it and the $A M S$ agent). In the case of the old LMaster not responding and the $A M S$ confirming that the old LMaster is gone, they accept the new LMaster. If the old LMaster responds to their messages, the message from the LMirror is ignored and team members continue to consider the old LMaster to be the leader of the team.

\subsection{Replication}

Thus far we have stated that when the LMirror is (re)created and when the LMaster is recreated, they 
are provided with all data necessary for team management. While this step is relatively obvious, the situation becomes somewhat more complicated afterward. The question is: how often data from the LMaster should be passed to the LMirror to be replicated. Note that the situation is different than in the case of a local replication (e.g. disk mirroring) as we have to assume that the LMaster and the LMirror reside on separate machines located in the Internet. One of possible approaches would be to replicate data each time any change occurs within the LMaster. This approach, however, is not likely to work for any large and active team. In this case number of information updates within the LMaster is likely to be large enough to lock both it and the LMirror. Note that each replication requires a confirmation that it was successfully completed. Only after such confirmation the LMaster would be able to continue its work. This mechanism is similar to what happens in the case of a standard database information replication procedure (with the difference that the database is usually located within a single data center).

The second possibility would be to send data to the LMirror and not wait for a confirmation. The danger is that some data will be lost (and we will have no control over what is actually replicated). In this way, over time the LMirror will contain only "random" data, which will not lead to correct re-creation of the LMaster.

Finally, it is possible to send updates in packages in specific time-intervals. When such a package is sent, the LMaster awaits confirmation of successful replication. Obviously, as a result of such procedure, recreation of the LMaster may result in creation of its somewhat outdated version (crash will happen sometime between replications), but at least data will be consistent up to the time of the last update. Therefore we assume that this approach is the best possible.

\section{Concluding remarks}

In this note we have presented our work devoted to development of an agent-based Grid resource-brokering system. We have focused on issues involved in team management. First, we have introduced the team-agentstatus monitoring tool, which we have implemented. This tool, being highly parameterized can be used as a centerpiece for adaptive team monitoring approach, which we are currently developing. We have also discussed processes involved in interactions between LMaster and LMirror agents, which are crucial for the team long-term survival. Across the note we have indicated a number of research directions that we plan to pursue. Another one of them, which was not mentioned thus far, is the necessary experimental work needed to establish the actual overhead of the proposed approach, especially in the case of a large team (note that ACL messaging introduces a number of overheads). We will report on our progress in subsequent reports.

\section{References}

[1] M. Dominiak, W. Kuranowski, M. Gawinecki, M. Ganzha, M. Paprzycki, Utilizing agent teams in Grid resource management-preliminary considerations. In: Proc. of the IEEE J. V. Atanasoff Conference, IEEE CS Press, Los Alamitos, CA, 2006, 46-51

[2] M. Dominiak, W. Kuranowski, M. Gawinecki, M. Ganzha, M. Paprzycki, Efficient Matchmaking in an Agent-based Grid Resource Brokering System, Proc. of the International Multiconference on Computer Science and Information Technology, PTI Press, 2006, 327-335

[3] FIPA Contract Net Interaction Protocol Specification, http://www.fipa.org/specs/fipa00029/ $\mathrm{SCOOO29H.html}$

[4] I. Foster, N. R. Jennings, C. Kesselman, Brain Meets Brawn: Why Grid and Agents Need Each Other, AAMAS'04, July, 2004, ACM Press, 2004, http://www. semanticGrid.org/documents/ 003-foster_i_Grid.pdf

[5] M. Dominiak, M. Ganzha, M. Gawinecki, W. Kuranowski, M. Paprzycki, S. Margenov, I. Lirkov, Utilizing Agent Teams in Grid Resource Brokering, International Transactions on Systems Science and Applications, 2007, in press

[6] M. Dominiak, M. Ganzha, M. Paprzycki, Selecting gridagent-team to execute user-job-initial solution, Proceedings of the Conference on Complex, Intelligent and Software Intensive Systems, IEEE CS Press, Los Alamitos, CA, 2007, 249-256

[7] M. Ganzha, M. Paprzycki, I. Lirkov, Trust Management in an Agent-based Grid Resource Brokering SystemPreliminary Considerations. In: M. Todorov (ed.), Applications of Mathematics in Engineering and Economics'33, American Institute of Physics, College Park, MD, 2007, 35-46

[8] JADE: Java Agent Development Framework. See http: $/ /$ jade.cselt.it

[9] W. Kuranowski, M. Paprzycki, M. Ganzha, M. Gawinecki, I. Lirkov, S. Margenov (2007) Agents as resource brokers in grids-forming agent teams. In: Proceedings of the LSSC Meeting, Springer (to appear)

[10] J. Dodgson, M. Spackman, A. Pearman, L. Phillips, DTLR multi-criteria analysis manual, UK: National Economic Research Associates, 2001

[11] H. Tianfield, R. Unland, Towards self-organization in multi-agent systems and Grid computing, Multiagent and Grid Systems, 1(2), 2005, 89-95

[12] D. Trastour, C. Bartolini, C. Preist, Semantic Web Support for the Business-to-Business E-Commerce Lifecycle, Proceedings of the International World Wide Web Conference, ACM Press, New York, USA, 2002, 89-98 